\title{
A Novel Small RNA Regulates Tolerance and Virulence in Shigella flexneri by Responding to Acidic Environmental Changes
}

\author{
Ligui Wang ${ }^{1,2 \dagger}$, Guang Yang ${ }^{1 \dagger}$, Lihua Qi ${ }^{1 \dagger}$, Xiang $\mathrm{Li}^{1}$, Leili Jia ${ }^{1}$, Jing Xie ${ }^{1}$, Shaofu Qiu ${ }^{1}$, \\ Peng $\mathrm{Li}^{1}$, RongZhang Hao ${ }^{1}$, Zhihao $\mathrm{Wu}^{1}$, Xinying $\mathrm{Du}^{1}$, Wuju $\mathrm{Li}^{2 *}$ and Hongbin Song ${ }^{1 *}$ \\ ${ }^{1}$ Institute of Disease Control and Prevention, Academy of Military Medical Sciences, Beijing, China, ${ }^{2}$ Center of \\ Computational Biology, Beijing Institute of Basic Medical Sciences, Beijing, China
}

Shigella flexneri is an important cause of bacillary dysentery in developing countries. Small regulatory RNAs (sRNAs) play essential roles in diverse cellular processes. We found a novel sRNA Ssr1 based on RT-PCR, northern blot, and 5'RACE in S. flexneri. Ssr1 responds to acidic environmental changes, as shown by a strong linear correlation between the $\mathrm{pH}$ value and Ssr1 expression $(R=0.785, P<0.05)$ using the qRT-PCR method. Deletion of Ssr1 results in growth retardation at $\mathrm{pH}$ values ranging from 5.0 to $7.0(P<0.05)$, and the survival rate was reduced by $22 \%$ in acidic conditions $(\mathrm{pH} 3.0)$. Additionally, virulence was significantly increased in an Ssr1 mutant strain, as revealed in a murine lung invasion model and survival model assays. By using the sTarPicker method and proteomic analysis, we considered that DnaK, which is a major factor that confers acidic stress tolerance, may be a direct target of Ssr1. We also found that Ssr1 may enhance virulence by directly targeting OmpA; this leads to altered expression of genes in the type three secretion system (T3SS). This work provides new insight into the mechanism of adaptation to environmental stress and into the pathogenesis of Shigella.

Keywords: sRNA, Shigella flexneri, response, stress tolerance, virulence

\section{INTRODUCTION}

Over the last few years, small regulatory RNAs (sRNAs)-based control mechanisms have been recognized as key regulators of gene expression. sRNAs vary from 50 to $500 \mathrm{nt}$ in length and are characterized by base pairing with target mRNAs; this affects the activity of the target mRNA or its translated protein product. sRNAs are generally un-translated and regulate diverse physiological processes in bacteria, such as stress responses, metabolism, and virulence, as well as the control of the bacterial envelope's composition (Storz et al., 2006; Toledo-Arana et al., 2007; Vogel, 2009). Surprisingly, only a few sRNAs have roles in both responding to environmental stresses and regulating virulence (Gripenland et al., 2010). For instance, an sRNA (ef0408-0409) mutant strain of Enterococcus faecalis was able to grow and survive more effectively than wild-type bacteria in the presence of osmotic and oxidative stress, and it was more resistant to acidic stress. Furthermore, the strain was more virulent (Michaux et al., 2014). In Shigella, RyhB sRNA is involved in the response to the environmental iron level, and it regulates the expression of the type three secretion system (T3SS), which is the major source of virulence (Murphy and Payne, 2007; Marteyn et al., 2012). 
Like other gram-negative enteric bacteria, Shigella encounter a gastric acidic $\mathrm{pH}(\mathrm{pH} 2-3)$ before reaching the colon after oral infection (Cheng et al., 2007). Shigella have the ability to survive in extreme environmental conditions and are pathogenic in host cells during the infection process. Thus, Shigella express a set of transcriptional regulators to adapt to and survive in different enteric environmental conditions such as low $\mathrm{pH}$, high temperature, and changing osmotic pressure (Murphy and Payne, 2007). Therefore, these bacteria have developed complex regulatory systems to respond to environmental signals (Papenfort and Vogel, 2014). Acidic stress adaptation confers resistance to a wide range of other stress conditions, including salinity, heat, and $\mathrm{H}_{2} \mathrm{O}_{2}$ (Cheng et al., 2007). This implies that the resistance to the gastrointestinal acidic environment is essential for pathogenesis in Shigella. Additionally, sRNAs are increasingly recognized as essential factors in the resistance to an acidic environment. As for an example, the GadY sRNA in Escherichia coli increases the expression of downstream acid resistance genes and regulates the GadX protein to increase the bacterial survival rate under low $\mathrm{pH}$ conditions (Opdyke et al., 2011).

Although a large number of Shigella strains have been sequenced, very little is known about sRNA identification and functions in this bacterium. Only nine sRNAs, $f f s, d s r A, m i c F$, $c s r B, g c v B, s s r S$, rnpS, spf, and oxyS, have been annotated in the Shigella genome using comparative genomic methods. In a recent study, nine novel sRNAs were identified and validated by computer-based methods and northern blot analyses, but the characteristics of these sRNAs remain to be elucidated (Peng et al., 2011). Only two sRNAs, RnaG, and RyhB, have been studied comprehensively in S. flexneri (Peng et al., 2011). The production of sRNAs that control bacterial virulence is required to finetune signaling and survive diverse environmental conditions. In particular, there have been few reports regarding the role of sRNAs in the response to environmental signals that increase tolerance to extreme host environments, and in the regulation of virulence. In this study, we identified a novel sRNA, Ssr1, during a comparative genomics bioinformatic screen and experimentally verified that it regulates virulence and the tolerance response to environmental acidity.

\section{MATERIALS AND METHODS}

\section{Bacterial Strains and Growth Media}

The strains used in this study are derivatives of the $S$. flexneri 2a 301 strain (the wild-type). S. flexneri strains were routinely cultured in LB medium ( $1 \%$ tryptone, $0.5 \%$ yeast extract and $1 \% \mathrm{NaCl}$ ) or on tryptic soy agar containing $0.01 \%$ (wt/vol) Congo red at $37^{\circ} \mathrm{C}$. When required, ampicillin, kanamycin, and chloromycetin were added to final concentrations of 100,50, and $30 \mu \mathrm{g} / \mathrm{mL}$, respectively.

\section{Construction of S. flexneri 301 sRNA1 Deletion Strain and the Complementation Strain}

The $\lambda$-Red-mediated recombination method was used to construct a $S$. flexneri 301 Ssr1 deletion mutant $(\Delta S s r 1)$ by replacing the $S s r 1$ gene with kanamycin resistance gene, encoding kanamycin resistance. Briefly, PCR was used to amplify regions of sequence upstream and downstream of the Ssrl gene using primer pairs (Park et al., 2001). Two $\sim 500$ bp sequences that overlapped the kanamycin resistance gene were fused by PCR with a complementary kanamycin resistance gene PCR fragment, resulting in the replacement of Ssr1 with kanamycin resistance gene. The resulting PCR product was gel purified using a gel extraction kit (Promega, A9285). The fused PCR fragment was transformed into the wild-type. $\Delta S s r 1$ were identified by screening transformants on LB agar plates containing kanamycin $(50 \mu \mathrm{g} / \mathrm{mL})$.

To construct the complementing plasmid pSsr1, the Ssr1 coding region and $200 \mathrm{bp}$ upstream of the transcriptional start site were amplified from the wild-type. These primers were designed to include unique restriction enzyme sites, $X b a \mathrm{I}$ and $S p h \mathrm{I}$, so that when the PCR amplicon was digested with XbaI and $S p h \mathrm{I}$, it could be ligated into a similarly digested plasmid, PACYC184, in an orientation-specific manner. Recombinant DNA products were verified by sequencing. The resulting plasmid was used to transform $\Delta S s r 1$ by electroporation, selecting for chloromycetin. The final complemented strain was called psRNA1.

\section{sRNA Prediction}

Given that sRNAs are mainly located in the intergenic regions of the genome, we predicted the promoter and terminator in the intergenic regions of Shigella flexneri. The profile search program pftools2.3 (Lesnik et al., 2001) was used for promoter prediction, and RNAMotif (Berg and von Hippel, 1987; Livny et al., 2005) was used for rho-independent terminator prediction. Only the intergenic regions with simultaneously predicted promoter and rho-independent terminator were chosen for experimental verification.

\section{RNA Isolation}

Cells were harvested from S. flexneri cultured at $37^{\circ} \mathrm{C}$ during mid-log phase $\left(\mathrm{OD}_{600}\right.$ of 0.4-0.6) by centrifugation. Total RNA from S. flexneri was prepared using the Trizol (Invitrogen, 15596108) procedure according to the manufacturer's instructions. RNA pellets were dissolved in DEPC- $\mathrm{H}_{2} \mathrm{O}$. Total nucleic acid concentrations and purity were estimated using absorbance readings $(260 \mathrm{~nm} / 280 \mathrm{~nm})$ on an Ultraspec II spectrophotometer.

\section{RT-PCR}

The total RNA was treated with DNase according to the manufacturer's instructions (Promega, M6101). No more than $10 \mu \mathrm{g}$ of total RNA was used to generate cDNA using a reverse transcription kit (Promega, K1005S) according to the product's directions. Each cDNA sample was diluted 1:10 in water, and $3 \mu \mathrm{L}$ was used as the template for each $25-\mu \mathrm{L}$ PCR. All probes were designed using Primer 5.0 (Listed as Table S1).

\section{Northern Blotting}

Northern blot analyses were carried out to confirm the transcription of sRNAs. A total of 18 candidate sRNAs were 
TABLE 1 | Small RNAs (sRNAs) in Shigella flexneri predicted in this study.

\begin{tabular}{|c|c|c|c|c|c|}
\hline sRNAgenes & Adjacent genes & Strand & $5^{\prime}$ end & $3^{\prime}$ end & RT-PCR \\
\hline Ssr1 & SF0268/yafV & $\rightarrow \rightarrow \leftarrow$ & 286,849 & $2,87,502$ & Yes \\
\hline Ssr2 & SF0490/SF0491 & $\leftarrow \rightarrow$ & 508,677 & $5,08,786$ & \\
\hline Ssr3 & ybfA/kdpA & $\leftarrow \rightarrow \rightarrow$ & 624,431 & $6,24,666$ & Yes \\
\hline Ssr4 & nagD/asnB & $\rightarrow \rightarrow \rightarrow$ & 652,524 & $6,52,614$ & \\
\hline Ssr5 & dacA/ybeD & $\rightarrow \rightarrow \rightarrow$ & 687,772 & $6,87,859$ & \\
\hline Ssr6 & SF4458/SF0680 & $\rightarrow \rightarrow \leftarrow$ & 712,385 & $7,12,787$ & Yes \\
\hline Ssr7 & ipaH_2/ybhE & $\leftarrow \rightarrow$ & 921,042 & $9,21,488$ & \\
\hline Ssr8 & SF0948/rlmL & $\leftarrow \rightarrow \rightarrow$ & 994,696 & $9,94,779$ & Yes \\
\hline Ssr9 & ymbA/fabA & $\rightarrow \rightarrow \leftarrow$ & $1,002,529$ & $1,002,752$ & Yes \\
\hline Ssr10 & icdA/SF1156 & $\rightarrow \rightarrow \rightarrow$ & $1,197,645$ & $1,198,294$ & Yes \\
\hline Ssr11 & SF1205/ychF & $\leftarrow \rightarrow \leftarrow$ & $1,252,826$ & $1,253,554$ & \\
\hline Ssr12 & pfkB/SF1508 & $\leftarrow \rightarrow \rightarrow$ & $1,540,588$ & $1,540,702$ & Yes \\
\hline Ssr13 & ydeJ/SF1559 & $\leftarrow \rightarrow \rightarrow$ & $1,591,049$ & $1,591,209$ & Yes \\
\hline Ssr14 & ribE/ydhE & $\leftarrow \rightarrow \rightarrow$ & $1,723,061$ & $1,723,156$ & \\
\hline Ssr15 & SF1779/tehB & $\leftarrow \rightarrow \leftarrow$ & $1,817,490$ & $1,817,786$ & \\
\hline Ssr16 & SF1897/SF4470 & $\rightarrow \rightarrow \leftarrow$ & $1,936,312$ & $1,936,490$ & \\
\hline Ssr17 & SF2011/insA & $\leftarrow \rightarrow \rightarrow$ & $2,036,426$ & $2,036,668$ & \\
\hline Ssr18 & yejH/rplY & $\rightarrow \rightarrow \rightarrow$ & $2,305,600$ & $2,305,712$ & \\
\hline Ssr19 & SF2423/vacJ & $\rightarrow \rightarrow \leftarrow$ & $2,474,415$ & $2,474,548$ & \\
\hline $\begin{array}{l}\text { Ssr20 } \\
\text { Ssr21 }\end{array}$ & $\begin{array}{l}\text { ddg/SF2445 } \\
\text { sseA/sseB }\end{array}$ & $\begin{array}{l}\rightarrow \rightarrow \leftarrow \\
\rightarrow \rightarrow \leftarrow\end{array}$ & $\begin{array}{l}2,499,782 \\
2,638,950\end{array}$ & $\begin{array}{l}2,500,097 \\
2,639,193\end{array}$ & \\
\hline $\begin{array}{l}\text { Ssr22 } \\
\text { Ssr23 }\end{array}$ & $\begin{array}{l}\text { SF2969/SF2970 } \\
\text { sap/SF2991 }\end{array}$ & $\begin{array}{l}\rightarrow \rightarrow \leftarrow \\
\rightarrow \rightarrow \rightarrow\end{array}$ & $\begin{array}{l}3,065,046 \\
3,087,241\end{array}$ & $\begin{array}{l}3,065,180 \\
3,087,450\end{array}$ & \\
\hline Ssr24 & SF3002/SF3003 & $\rightarrow \rightarrow \rightarrow$ & $3,099,131$ & $3,099,293$ & \\
\hline Ssr25 & ygjR/ygjT & $\rightarrow \rightarrow \rightarrow$ & $3,228,567$ & $3,228,805$ & \\
\hline Ssr26 & SF3873/hemY & $\leftarrow \leftrightarrow$ & $3,994,443$ & $3,994,974$ & Yes \\
\hline Ssr27 & engB/yihl & $\leftarrow \rightarrow \rightarrow$ & $4,063,618$ & $4,063,972$ & \\
\hline Ssr28 & yjdA/phnA & $\leftarrow \rightarrow \rightarrow$ & $4,281,467$ & $4,281,794$ & Yes \\
\hline Ssr29 & SF4216/SF4217 & $\rightarrow \rightarrow \rightarrow$ & $4,390,809$ & $4,390,927$ & \\
\hline Ssr30 & dcuAvaspA & $\leftarrow \rightarrow \leftarrow$ & $4,470,403$ & $4,470,496$ & \\
\hline Ssr31 & pagP/dcuC & $\leftarrow \leftarrow \rightarrow$ & $6,94,274$ & $6,93,980$ & Yes \\
\hline Ssr32 & SF4458/SF0680 & $\rightarrow \leftarrow \leftarrow$ & $7,12,535$ & $7,12,712$ & \\
\hline Ssr33 & mdoH/SF1046 & $\rightarrow \leftarrow \leftarrow$ & $1,090,831$ & $1,091,008$ & \\
\hline Ssr34 & SF1350/insB & $\rightarrow \leftarrow \leftarrow$ & $1,397,476$ & $1,398,217$ & \\
\hline Ssr35 & SF1542/ycgW & $\rightarrow \leftarrow \rightarrow$ & $1,576,030$ & $1,576,603$ & \\
\hline Ssr36 & ycgW/SF4467 & $\rightarrow \leftarrow \leftarrow$ & $1,577,114$ & $1,577,901$ & \\
\hline Ssr37 & ydeJ/SF1559 & $\leftarrow \leftarrow \rightarrow$ & $1,591,802$ & $1,591,911$ & Yes \\
\hline Ssr38 & ydbK/SF1823 & $\rightarrow \leftarrow \rightarrow$ & $1,860,960$ & $1,861,117$ & \\
\hline Ssr39 & SF1879/ipaH_4 & $\rightarrow \leftarrow \rightarrow$ & $1,917,499$ & $1,917,778$ & \\
\hline Ssr40 & SF1927/SF1928 & $\rightarrow \leftarrow \rightarrow$ & $1,963,576$ & $1,963,685$ & \\
\hline Ssr41 & yecl/SF1950 & $\rightarrow \leftarrow \rightarrow$ & $1,984,123$ & $1,984,327$ & Yes \\
\hline Ssr42 & nmpC/SF1978 & $\rightarrow \leftarrow \leftarrow$ & $2,010,014$ & $2,010,161$ & \\
\hline Ssr43 & SF2038/SF4477 & $\leftarrow \leftarrow \leftarrow$ & $2,060,687$ & $2,060,776$ & \\
\hline Ssr44 & SF2042/SF2043 & $\leftarrow \leftarrow \leftarrow$ & $2,063,884$ & $2,064,349$ & Yes \\
\hline Ssr45 & $\mathrm{fadl} / \mathrm{fadL}$ & $\leftarrow \leftarrow \rightarrow$ & $2,469,554$ & $2,469,983$ & Yes \\
\hline Ssr46 & SF2493/SF2494 & $\rightarrow \leftarrow \rightarrow$ & $2,554,471$ & $2,554,606$ & \\
\hline Ssr47 & sseA/sseB & $\rightarrow \leftarrow \leftarrow$ & $2,638,501$ & $2,638,972$ & Yes \\
\hline Ssr48 & $\mathrm{rpsP} / \mathrm{ffh}$ & $\leftarrow \leftarrow \leftarrow$ & $2,744,850$ & $2,744,947$ & \\
\hline Ssr49 & stpA/SF2698 & $\leftarrow \leftarrow \rightarrow$ & $2,772,656$ & $2,772,940$ & \\
\hline Ssr50 & insA/yghK & $\leftarrow \leftarrow \leftarrow$ & $3,107,699$ & $3,106,244$ & \\
\hline Ssr51 & SF3060/sufl & $\leftarrow \leftarrow \leftarrow$ & $3,156,062$ & $3,156,664$ & Yes \\
\hline
\end{tabular}

(Continued)
TABLE 1 | Continued

\begin{tabular}{llllll}
\hline sRNAgenes & Adjacent genes & Strand & $\mathbf{5}^{\prime}$ end & $\mathbf{3}^{\prime}$ end & RT-PCR \\
\hline Ssr52 & greA/dacB & $\leftarrow \leftarrow \rightarrow$ & $3,320,988$ & $3,321,087$ & \\
Ssr53 & yhhX/yhhY & $\leftarrow \leftarrow \rightarrow$ & $3,553,565$ & $3,553,647$ & \\
Ssr54 & hdeD/yhiE & $\rightarrow \leftarrow \rightarrow$ & $3,635,036$ & $3,635,153$ & Yes \\
Ssr55 & yhiX/yhiW & $\rightarrow \leftarrow \rightarrow$ & $3,698,133$ & $3,698,286$ & \\
Ssr56 & shiD/insB & $\rightarrow \leftarrow \leftarrow$ & $3,817,761$ & $3,817,881$ & \\
Ssr57 & yijP/ppc & $\leftarrow \leftarrow \leftarrow$ & $4,165,277$ & $4,165,400$ &
\end{tabular}

tested by northern blotting. Total RNA $(15-20 \mu \mathrm{g})$ was separated by electrophoresis in a $10 \%$ polyacrylamide gel and transferred to a nylon membrane by electroblotting. RNAs were crosslinked to the membrane by exposure to ultraviolet light (Thermo Scientific, 89880). The membranes were hybridized with gene-specific biotin-labeled oligonucleotides, and hybridization signals were visualized using a Phosphor Imager (Molecular Dynamics).

\section{RACE}

RACE experiments were performed according to the manufacturer's instructions (Takara Biochemicals, D315) to identify the $5^{\prime}$ ends of the cDNAs of interest. This method allows the discrimination of $5^{\prime}$ ends generated by transcription start sites and end sites.

\section{Stress Tolerance Assays}

For growth experiments, overnight cultures grown in LB medium or LB medium containing kanamycin were diluted 1:100 in LB with the appropriate supplement and grown at $37^{\circ} \mathrm{C}$ with shaking at $160 \mathrm{rpm} / \mathrm{min}$. For stress tolerance assays, the medium was adjusted to a specific $\mathrm{pH}$ level, 5.0, 6.0, 7.0, or 8.0. Bacterial growth was monitored by measuring the $\mathrm{OD}_{600}$.

For stress tolerance assays, the wild-type and $\Delta S s r 1$ were exposed to in vitro environmental stress conditions. Bacteria were inoculated into LB medium and grown to the early logarithmic phase $\left(\mathrm{OD}_{600}\right.$ of $\left.0.6-0.8\right)$ at $37^{\circ} \mathrm{C}$. To determine the response to acidic stress, each strain was incubated at $37^{\circ} \mathrm{C}$ for $30 \mathrm{~min}$ in $\mathrm{LB}$ medium at $\mathrm{pH}$ 3.0. After the treatment, cells were diluted and plated on LB to determine the number of CFUs. Results represent the mean of at least three separate experiments.

\section{Sereny Test}

The sereny keratoconjunctivitis test was performed as described previously (Sereny, 1955) to evaluate the virulence of the wildtype and $\Delta S s r 1$. Overnight bacterial cultures were serially diluted to suitable $\mathrm{CFU} / \mathrm{mL}$ in $\mathrm{NaCl}$ for infection. A $20-\mu \mathrm{L}$ drop of a $10^{8}$ $\mathrm{CFU}$ concentration was injected into the conjunctival sac of each guinea pig's right eye, and the left eye was injected with $\mathrm{NaCl}$ as a control ( $n=5$ mice in each group). Guinea pigs were observed at 24,48 , and $72 \mathrm{~h}$ after inoculation for signs of infection and inflammation in their eyes and assigned scores as follows: "-" for normal eye indistinguishable from the contralateral uninoculated eye, "+" for lacrimation or eyelid edema, " ++ " for lacrimation or eyelid edema plus mild conjunctival hyperemia, " +++ " for 
TABLE 2 | Keratoconjunctivits in guinea pigs inoculated with wild-type and $\Delta S s r 1$, as well as a $\mathrm{NaCl}$ control.

\begin{tabular}{|c|c|c|c|c|c|c|c|c|c|c|c|c|c|c|c|}
\hline \multirow{3}{*}{ species } & \multicolumn{15}{|c|}{ Time } \\
\hline & \multicolumn{5}{|c|}{$24 \mathrm{~h}$} & \multicolumn{5}{|c|}{$48 \mathrm{~h}$} & \multicolumn{5}{|c|}{$72 \mathrm{~h}$} \\
\hline & 1 & 2 & 3 & 4 & 5 & 1 & 2 & 3 & 4 & 5 & 1 & 2 & 3 & 4 & 5 \\
\hline Control & - & - & - & - & - & - & - & - & - & - & - & - & - & - & - \\
\hline Wild-Type & + & + & + & + & + & ++ & ++ & ++ & ++ & ++ & +++ & +++ & +++ & ++++ & ++++ \\
\hline$\Delta S s r 1$ & ++ & ++ & ++ & ++ & ++ & +++ & +++ & +++ & +++ & +++ & ++++ & ++++ & ++++ & ++++ & ++++ \\
\hline
\end{tabular}

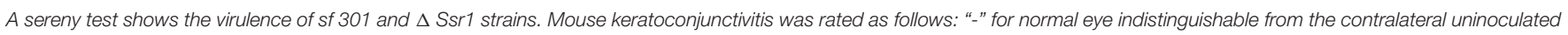

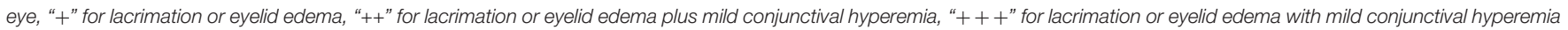
plus slight exudates, and " ++++ " for full-blown purulent keratoconjunctivitis.

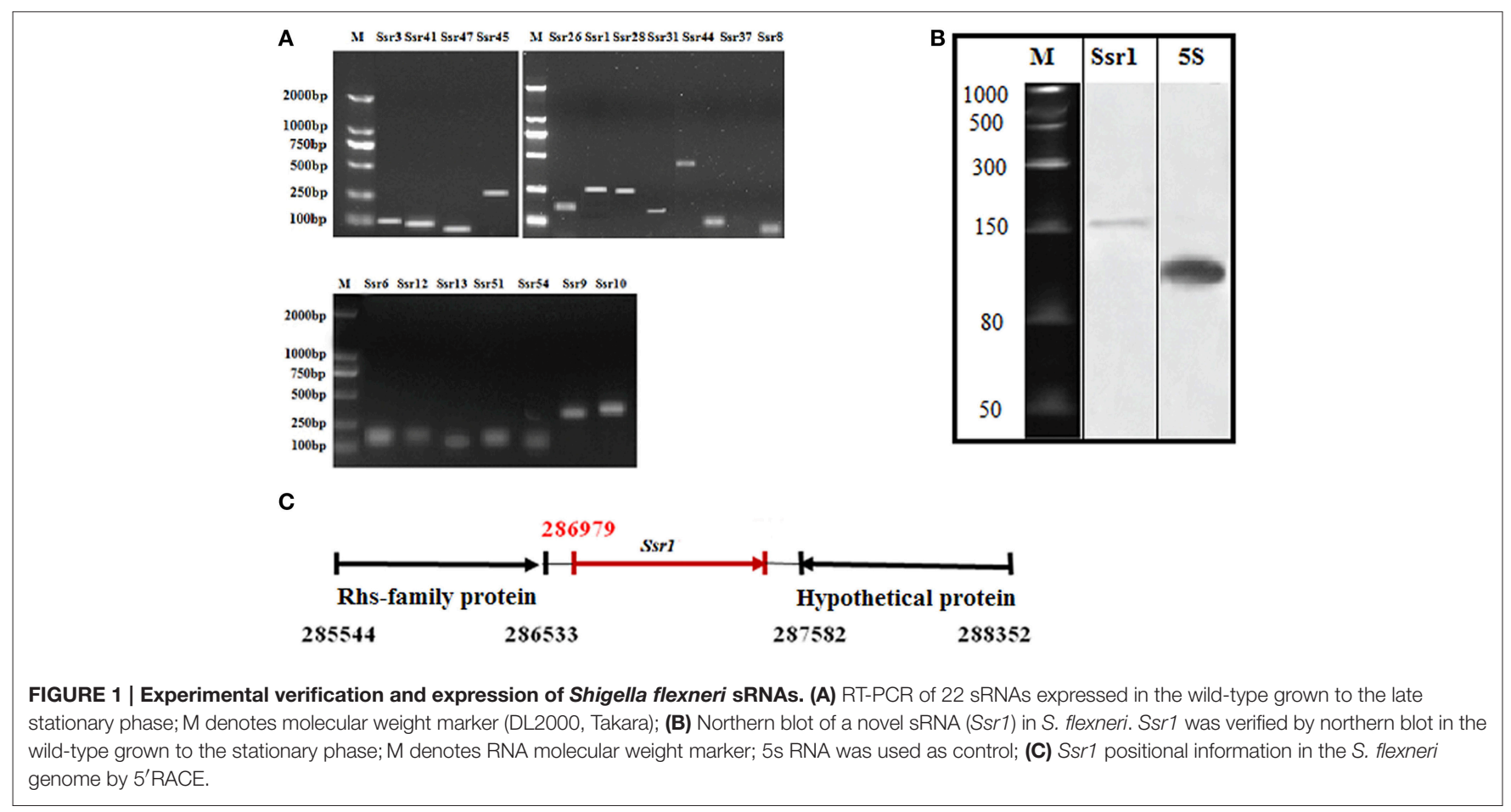

lacrimation or eyelid edema with mild conjunctival hyperemia plus slight exudates, and “ ++++ " for full-blown purulent keratoconjunctivitis.

\section{Mouse Infection}

Six-week-old Balb/c female mice weighing 20 g obtained from the Animal Center of the Academy of Military Medical Sciences were anesthetized by diethyl ether. A bacterial suspension of $20 \mu \mathrm{L}$ was applied intranasally to each mouse with a pipette. A group of eight mice were challenged with $10^{6}$ CFUs. Lungs were collected from all animals $24 \mathrm{~h}$ after infection, washed with PBS to remove contaminating blood, and homogenized. The wild-type and $\Delta S s r 1$ samples were serially diluted and the resulting colonies were counted on Brain Heart Infusion plates with and without kanamycin. Results were recorded by the competition index. For survival studies, the wild-type and $\Delta S s r 1$ were introduced intranasally at $10^{6}$ CFUs. Ten mice per group were used in these studies. Mice were monitored daily for survival. The statistical analysis was performed using the log-rank (Mantel-Cox) test.

\section{sRNA Targets Prediction}

The sTarPicker prediction method was used to predict the target mRNAs of ssr1 against the entire genome of the wild-type (Vandal et al., 2009). This genome-wide prediction application is available at http://ccb.bmi.ac.cn/starpicker/prediction.php. The program is based on a two-step model of hybridization between an sRNA and a target. In comparisons with different sRNA target prediction tools, such as IntaRNA, TargetRNA, and sRNATarget, we found that sTarPicker performed best in both the accuracy of predicted binding sites and in identification of sRNA targets on an independent test dataset (Ying et al., 2011).

A 2-DE analysis was performed according to previously described methods with slight modifications (Li et al., 2011; Zhou et al., 2011). In brief, the prepared pooled protein samples $(600 \mathrm{mg}$ protein on preparative gels or $120 \mathrm{mg}$ protein on 


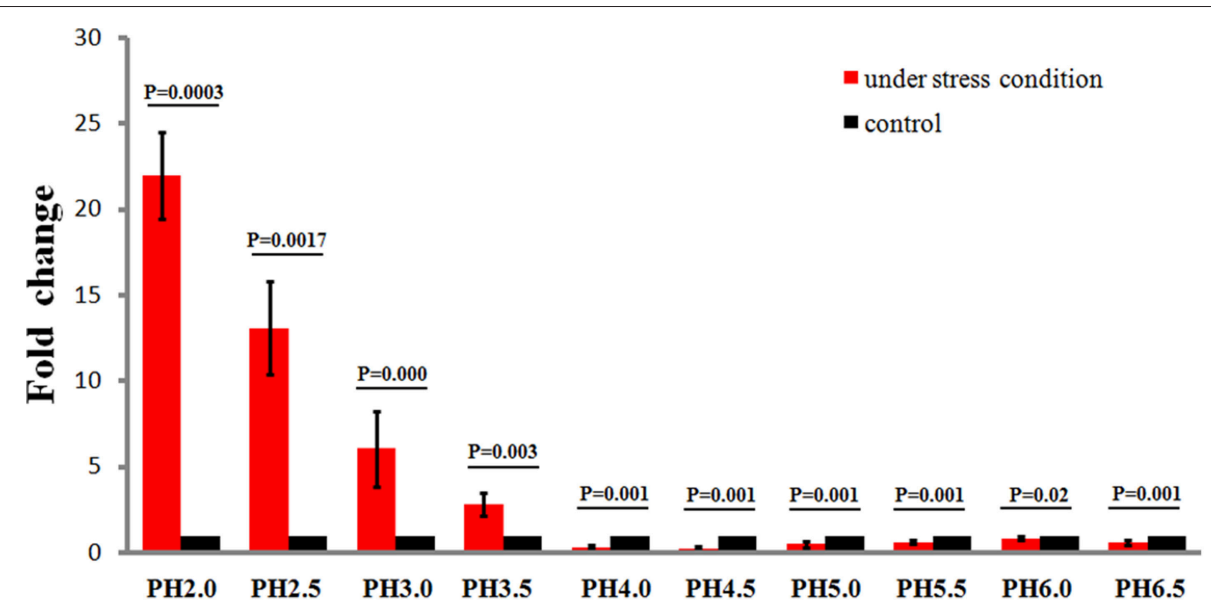

FIGURE 2 | Relative transcription of the sRNA Ssr1 in the wild-type when exposed to a range of pH conditions for $\mathbf{3 0}$ min. The wild type was cultured in $\mathrm{LB}$ broth buffered to a range of $\mathrm{pH}$ values. Expression of Ssr1: red bars indicate fold changes calculated as means from triplicate experiments, representing the ratios of Ssr1 expression levels under various $\mathrm{pH}$ conditions compared with $\mathrm{pH}$ 7.0. Standard deviations are indicated by the error bars. Black bars represent the control transcript values. The statistical analysis was performed using the Student's $t$-test.
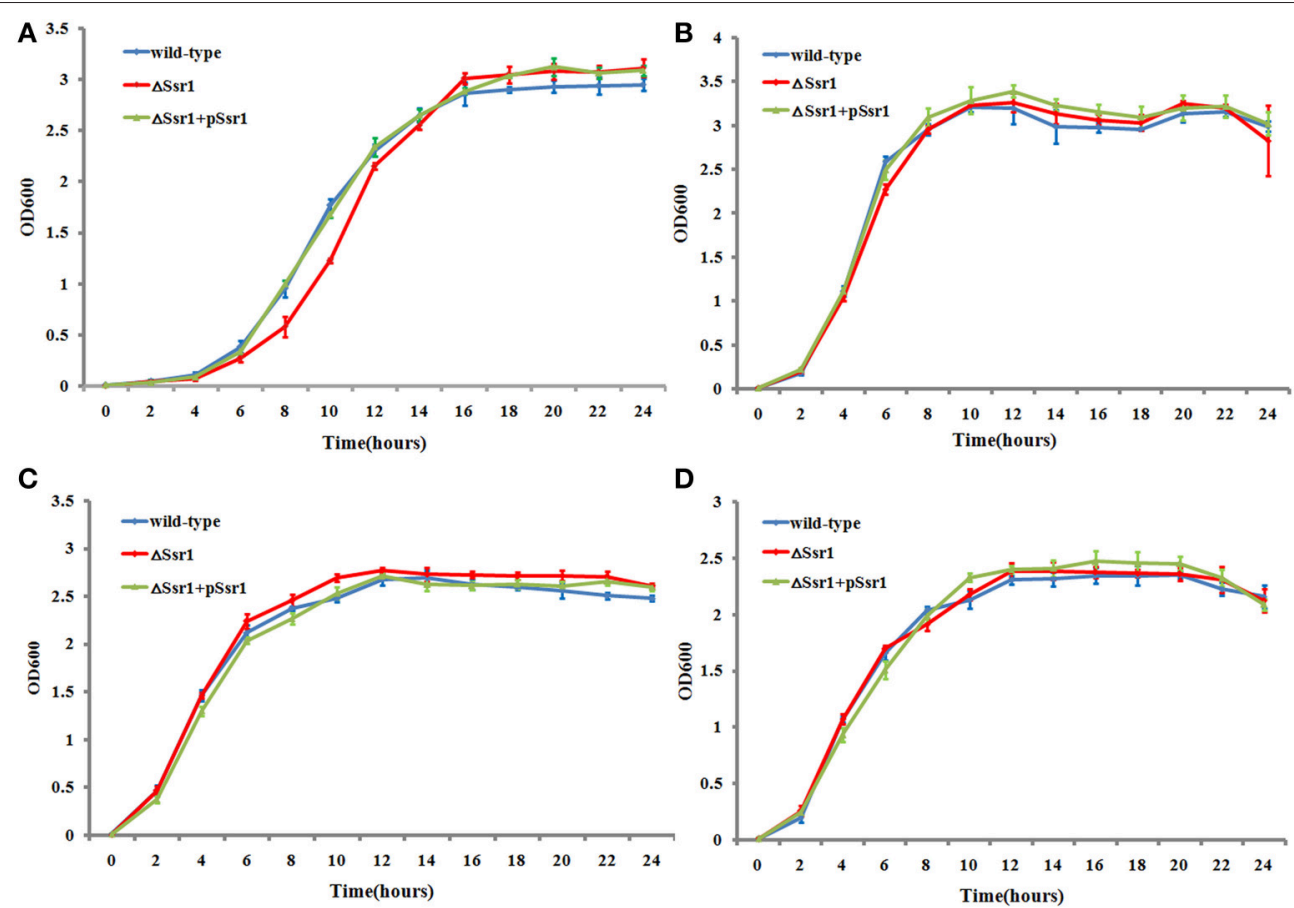

FIGURE 3 | Acidic stress tolerance of Shigella flexneri. Growth characteristics of the wild-type, $\Delta S s r 1$, and complementation strains in LB under different pH levels: (A) pH 5.0; (B) $\mathrm{pH}$ 6.0; (C) $\mathrm{pH}$ 7.0; and (D) $\mathrm{pH}$ 8.0. The error bars indicate standard deviations based on duplicate experiments.

analytical gels) were mixed with rehydration buffer to a volume of $450 \mathrm{~mL}$. The IPG strips ( $\mathrm{pH} 4-7,24 \mathrm{~cm}$, GE Healthcare, $17-$ 6002-46) for the first dimension were used to isolate the altered proteins, and the running condition was set at $20^{\circ} \mathrm{C}$, step 1 : $300 \mathrm{~V}$ for $0.5 \mathrm{~h}$, step $2: 700 \mathrm{~V}$ for $0.5 \mathrm{~h}$, step $3: 1500 \mathrm{~V}$ for $1.5 \mathrm{~h}$, step 4: $9900 \mathrm{~V}$ for $3 \mathrm{~h}$, step 5: $9900 \mathrm{~V}$ for $6.5 \mathrm{~h}$, step 6: $600 \mathrm{~V}$ for $20 \mathrm{~h}$, and step 7: $8000 \mathrm{~V}$ constant for a total of 56,000 Vh. After completion of the isoelectric focusing program, the strips were equilibrated in two steps: $15 \mathrm{~min}$ in an immobilized $\mathrm{pH}$ gradient equilibration buffer [ $6 \mathrm{M}$ urea, $2 \%$ SDS, $30 \%$ glycerol, $0.375 \mathrm{M}$ Tris ( $\mathrm{pH} 8.8), 20 \mathrm{mg} / \mathrm{mL}$ DTT, and a trace of bromophenol blue] and then alkylated for $15 \mathrm{~min}$. Subsequently, a $12.5 \%$ SDS-PAGE 2-DE was performed. Electrophoresis was carried out at $20 \mathrm{~mA}$ per gel for $40 \mathrm{~min}$ and then at $30 \mathrm{~mA}$ per gel until the dye front reached the bottom. The protein spots were visualized via either silver staining or Coomassie Brilliant Blue 
G-250 staining. Triplicate 2-DE gels were performed for each group.

Triplicate gels from $\Delta S s r 1$ and wild type strain (control) were analyzed for spot intensity using Image Master 2D Platinum software (GE Healthcare, 28-9408-30) according to the protocols provided by the manufacturer. The criterion for significant changes in protein expression was a difference of at least 1.5 -fold between the $\Delta S s r 1$ and wild type strain groups.

\section{Protein Identification}

The protein identification was performed according to the method described by Shi et al. (2009). Gel spots showing significant changes were excised from 2-DE gels. Gel spots were washed and then digested with sequencing-grade trypsin. MALDI-TOF MS and TOF/TOF tandem MS were performed on a MALDI-TOF-TOF mass spectrometer (4800 Proteomics Analyzer, Applied Biosystems,). The instrument was set in

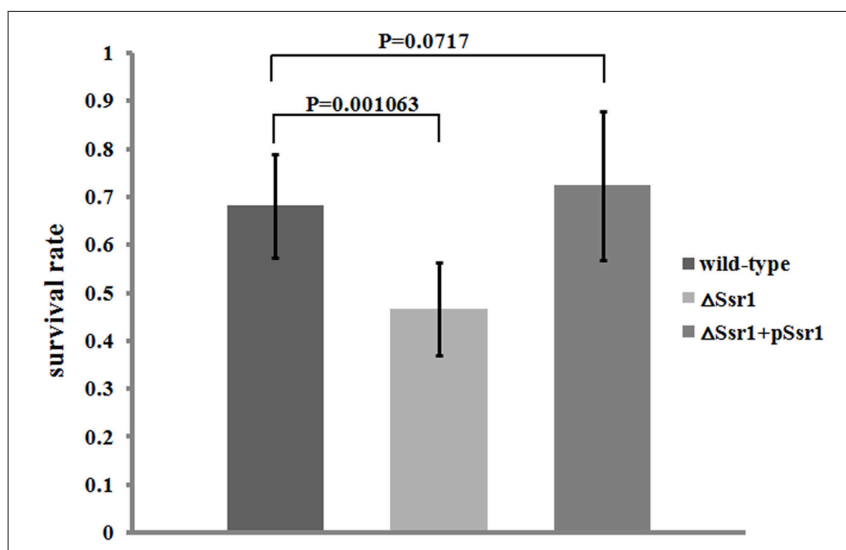

FIGURE 4 | Survival of $\Delta S s r 1$ relative to the wild-type when exposed to acid stresses. Wild-type, $\Delta S s r 1$, and complementation $(\Delta S s r 1+p S s r 1)$ strains were grown in $\mathrm{LB}(\mathrm{pH} 7.0)$ to the exponential phase and then subjected to acidic stress ( $\mathrm{pH}$ 3.0) conditions. Recovered colony-forming units were determined by counting plated serial dilutions as described in the Materials and Methods. Bars represent the mean percent survival compared with untreated controls. Each assay was conducted in three replicates. A statistical analysis was performed using the Student's t-test. reflector mode. Peptide mass fingerprints coupled with peptide fragmentation patterns were used to identify the protein in the International Protein Index (IPI) database using the MASCOT search engine. The functions of the identified proteins and their associated biological processes were matched by searching Gene Ontology (http://www.geneontology.org).

\section{Ethics Statement}

The animals were obtained from the laboratory animal center (Academy of Military Medical Sciences). The methods were carried out in accordance with the approved guideline of Academy of Military Medical Sciences. The experimental protocol was approved by the Ethics Committee for Animal Experimentation of the Academy of Military Medical Sciences.

\section{RESULTS}

\section{Novel Small Regulatory RNA (sRNA) in S. flexneri}

Using a transcription unit-based method, we predicted 57 sRNAs in the intergenic regions of the S. flexneri 2a 301 strain (the wild-type) (NC_004337). These predicted sRNAs are referred to hereafter as "Ssrs" (for Shigella small RNAs). The sRNAs varied from 50 to $500 \mathrm{nt}$ in length and they were numbered from 1 to 57 (Table 1). To test whether all these regions express detectable transcripts, RT-PCR was performed, and 18 sRNAs were then validated (Figure 1A). According to its high level of expression in S. flexneri, we opted to perform northern blot for Ssr1, which revealed that the length of small RNA (Ssr1) was $\sim 150$ bp (Figure 1B). We conducted homology analysis in other enteric bacteria, and found that Ssr1 only exists in Shigella.

We determined the transcriptional start site of $S s r 1$ by using rapid amplification of $5^{\prime} \mathrm{cDNA}$ ends (5'RACE) analysis. $5^{\prime} \mathrm{RACE}$ analysis identified the Ssr1 $5^{\prime}$ end, which is located at 286979 (Figure 1C). Ssr1 is located in an intergenic region between two open reading frames, SF0268 (encoding a putative Rhsfamily protein) and SF0269 (encoding a hypothetical protein). To investigate the role of $S s r 1$ in $S$. flexneri, a mutant, $\Delta S s r 1$, was derived from the wild-type by deleting the $S s r 1$ sequence and replacing it with a kanamycin resistance sequence. Additionally,

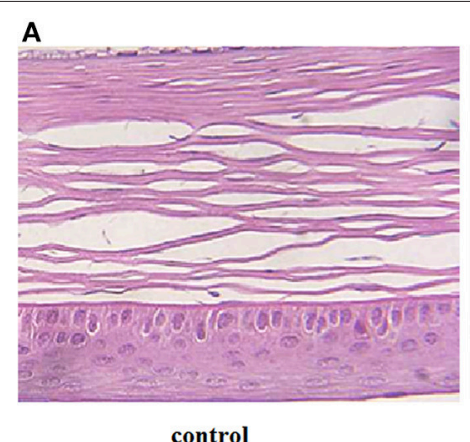

control

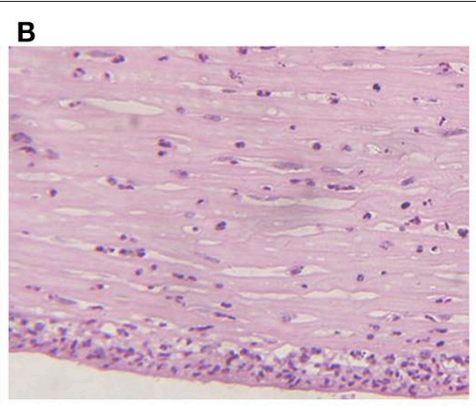

wild-type

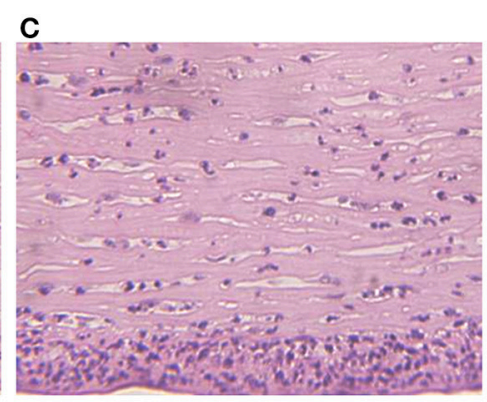

$\Delta \mathrm{Ssr} 1$

FIGURE 5 | The corneal pathology of the Shigella flexneri wild-type and $\mathbf{\Delta}$ Ssr1. Inflammatory cells are more in $\Delta$ Ssr 1 than in the wild-type strain, while the $\mathrm{NaCl}$ control group has no inflammatory cells. (A) In the blank group (B) the wild-type group (C) the mutant $\Delta S s r 1$ strain. 
an Ssr1 complementation strain was constructed by transforming the plasmid pACYC184 containing the Ssr1 gene into the Ssr1 mutant strain to assess the complementation of function in trans.

\section{Ssr1 Is a Novel Factor Involved in the Response to Acidic Stress}

To study the effects of different acidic stress conditions on Ssr1, we exposed S. flexneri to media of different pH. qRT-PCR revealed that $S s r 1$ was most highly expressed in the $\mathrm{pH} 2.0$ 4.0 range. The highest expression was at $\mathrm{pH} 2.0$ compared with the expression level at $\mathrm{pH} 7.0$, and expression significantly declined from $\mathrm{pH} 4.5$ to 6.5 (Figure 2). Interestingly, a strong linear correlation between $\mathrm{pH}$ value and $S$ srl expression was observed $(R=0.785, P<0.05)$. These results suggest that Ssr1 may be highly expressed in the stomach of the host $(\sim \mathrm{pH} 1.5-3.5)$ during an S. flexneri infection. Thus, Ssr1 expression may be regarded as a response to an acidic environmental change as the bacterium colonizes the gastrointestinal tract.

\section{Reduced Stress Tolerance and Survival of the S. flexneri Ssr1 Mutant}

The ability of enteric bacteria to thrive in the extremely acidic environment of the stomach is crucial for colonization and survival in the intestine (Hoe et al., 2013). To investigate these functions of $S s r 1$, we successfully created $\Delta S s r 1$ and a plasmid-based complementation strain of $S s r 1$. The effects of $S s r 1$ on $S$. flexneri growth in acidic media were then examined. $\Delta S s r 1$ grown in medium adjusted to $\mathrm{pH} 5.0$ exhibited a significantly prolonged lag in the exponential phase compared with the wildtype $(P<0.05)$ (Figure 3A). Under $\mathrm{pH} 6.0,7.0$, and 8.0, $\Delta S s r 1$ entered exponential phase at $2 \mathrm{~h}$ and entered a stationary phase at $10 \mathrm{~h}$, with the wild-type exhibiting the same growth tendency (Figures 3B-D). There was no significant difference between the growth of the wild-type and the Ssr1 complementation strains under the above conditions.

S. flexneri can survive various stresses, including that engendered by low pH (Teixeira-Gomes et al., 2000). To further determine the role of Ssr1 in the acidic stress tolerance of $S$. flexneri, the survival rates of $\Delta S s r 1, S s r 1$ complementation, and the wild-type during acidic stress ( $\mathrm{pH}$ 3.0) were compared. This revealed that the survival rate of $\Delta S s r 1$ decreased by $22 \%$ under low $\mathrm{pH}$ stress $(P<0.05)$, and the complementation strain exhibited no significant difference (Figure 4). This result indicated that Ssr1 in $S$. flexneri plays an important role in the resistance to acidic stress.

\section{The Novel Ssr1 Regulates Virulence in S. flexneri}

To determine whether $S s r 1$ is important for virulence, guinea pig conjunctival sacs were injected with $\Delta S s r 1$ and the wildtype. Guinea pigs inoculated with the wild-type developed slight conjunctivitis without purulence at $24 \mathrm{~h}$ post-infection, which developed to keratoconjunctivitis with purulence after $48 \mathrm{~h}$, and continued to be severe at $72 \mathrm{~h}$. Guinea pigs inoculated with $\Delta S s r 1$ displayed a severe keratoconjunctivitis with purulence

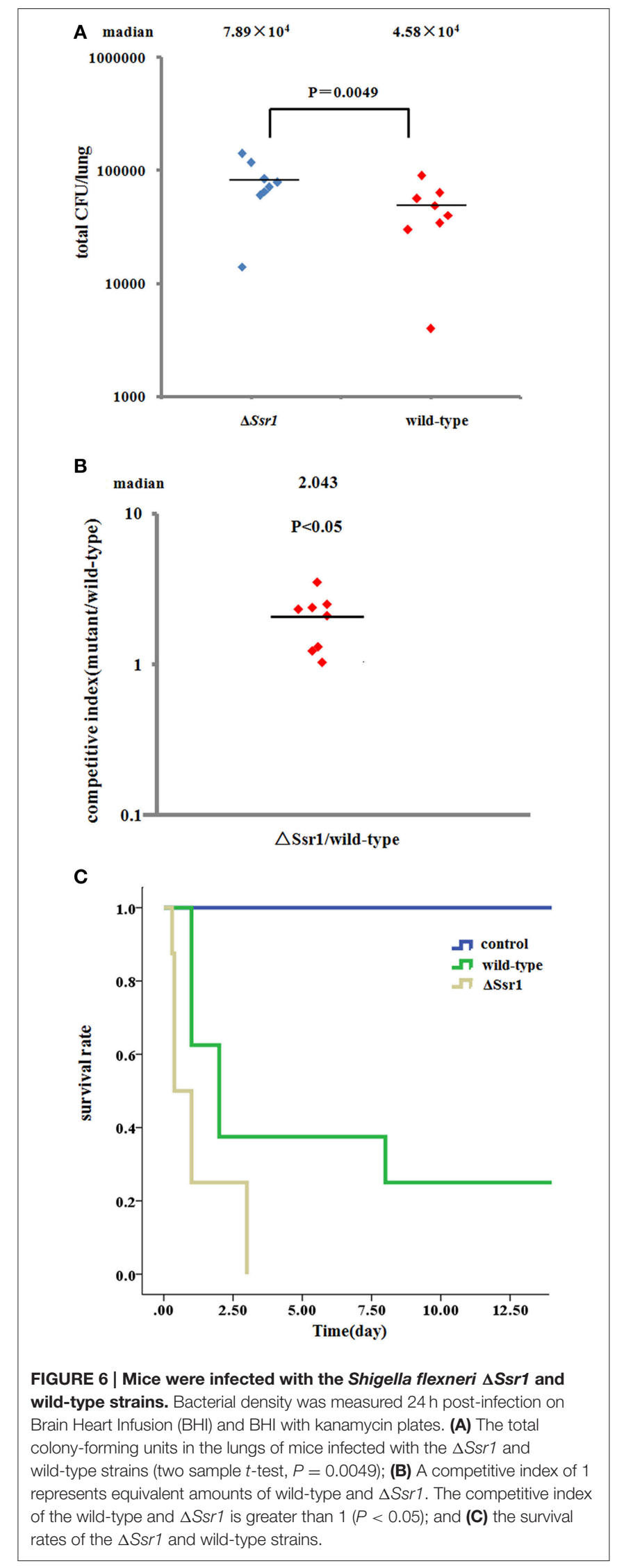


at $24 \mathrm{~h}$, and at 48 and $72 \mathrm{~h}$, the situation was increasingly severe. The $\mathrm{NaCl}$ control group did not develop conjunctive inflammation (Table 2). Then, the eyes of the experimental guinea pigs were analyzed by biopsy, and hematoxylin and eosin staining. The eyes of guinea pigs infected with both the wild-type and mutant showed an inflammatory reaction such as corneal epithelial cell necrosis, shedding, and corneal intrinsic membrane fiber disorder, accompanied by bleeding and inflammatory cell infiltration. But the inflammation of the guinea pigs carrying $\Delta S s r 1$ was more serious than that of infected with the wild-type (Figure 5). The $\mathrm{NaCl}$ control group elicited no inflammatory response in this test. Together, these results suggest that $S s r 1$ expression may be related to the virulence of S. flexneri.

To further confirm the keratoconjunctivitis results, the wildtype and $\Delta S s r 1$ were tested in a mouse lung invasion assay. The number of total $\Delta S s r 1$ colony-forming units (CFUs) recovered after gentamicin treatment to a mean value of $7.89 \times 10^{4}$. This was significantly higher $(P=0.0049)$ than the number of the wildtype CFUs $\left(4.58 \times 10^{4}\right)$ (Figure 6A). The calculated confidence interval median value was 2.043. This strongly suggests that $\Delta S s r 1$ has an impact on the capability of S. flexneri to effectively invade cultured cells (Figure 6B).

To further determine the pathogenic role of Ssr1 in vivo, $\mathrm{BALB} / \mathrm{c}$ mice were infected intranasally with the wild-type and $\Delta S s r 1$, and survival was monitored. Survival rates of $62.5 \%(5 / 8$ mice) infected with the wild-type and 25\% (2/8 mice) infected with $\Delta S s r 1$ were observed at 1 day post-infection (log-rank test, $P=0.00)$. At 3 days post-infection, the group infected with $\Delta S s r 1$ had a mortality rate of $100 \%(8 / 8)$, while the mice infected with the wild-type strain had a mortality rate of $62.5 \%(5 / 8)$ (Figure 6C).

\section{Identification of Ssr1 Targets}

sRNAs usually regulate other genes at a post-transcriptional level by directly or indirectly interacting with the associated mRNA.
To identify the targets of Ssr1 sRNA, we used sTarPicker to search the regions of $S$. flexneri mRNAs for potential RNA duplex formation with Ssr1. This analysis predicted 283 binding sites and suggested that Ssr1 sRNAs possible interact directly with these target mRNAs via a common region. Subsequently, the two-dimensional gel electrophoresis (2-DE) technique was used to compare $\Delta S s r 1$ to the wild-type to confirm these targets. For the proteomic analysis, total proteins were harvested during the stationary phase of bacteria. Protein spots with a change of more than 1.5-fold were selected for further analysis. The 2-DE maps of the wild-type and $\Delta S s r 1$ are shown in Figure 7. Detailed information on the up- and downregulated proteins is listed in Table 3.

Fifty-one differentially expressed proteins were successfully identified. Among them 24 proteins were upregulated and 27 proteins were downregulated in $\Delta S s r 1$ (Table 3). The identified proteins were mainly involved in amino acid transport and metabolism, cell wall, carbohydrate transport and metabolism, and energy production. The proteins had diverse cellular locations, including the cytoplasm and cell membranes, while others were secreted, and participate in various metabolic pathways that are regulated by sRNAs, suggesting that Ssr1 has a role in the modulation of bacterial colonization and pathogenicity. The level of a stress response protein (DnaK) was increased 1.92-fold compared with $\Delta S s r 1$. DnaK is upregulated in order to protect cells from several stress conditions (Tomoyasu et al., 2012). Another important protein is the OmpA, which was increased 1.80 -fold compared with $\Delta S s r 1$. The downregulated proteins included those involved in the T3SS response compared with $\Delta S s r 1$. IpaA, ipaD, ipgC, and mxiC were downregulated $7.76,3.39,5.56$, and 4.36-fold, respectively. These T3SS factors are indispensable for virulence in S. flexneri. Using qRT-PCR, we analyzed the expression of six genes at $\mathrm{pH}=7.0$ (outer membrane protein OmpA, the general stress response molecular chaperone Dnak, and IpaA, IpaD, IpgC, and MxiC; these last four genes belong to the T3SS and are thus important in virulence).

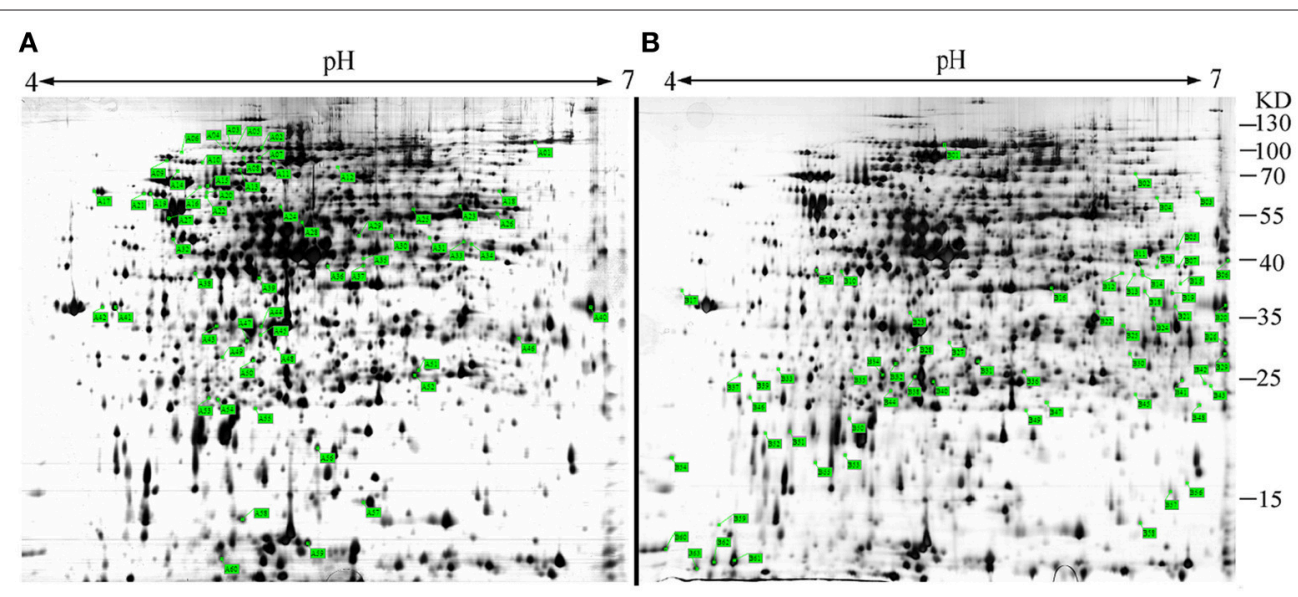

FIGURE 7 | Representative two-dimensional gel electrophoresis of the wild-type and $\mathbf{\Delta}$ Ssr1. The two figures represent differential protein expression levels in the wild-type (A) and $\Delta S s r 1$ (B) in the stationary phase. The highlighted spots in (A) represent proteins that have greater expression levels in the wild-type, whereas those in (B) represent proteins that are more abundant in the $\Delta S s r 1$. 
TABLE 3 | Differentially expressed proteins in $\Delta$ Ssr1-Shigella flexneri.

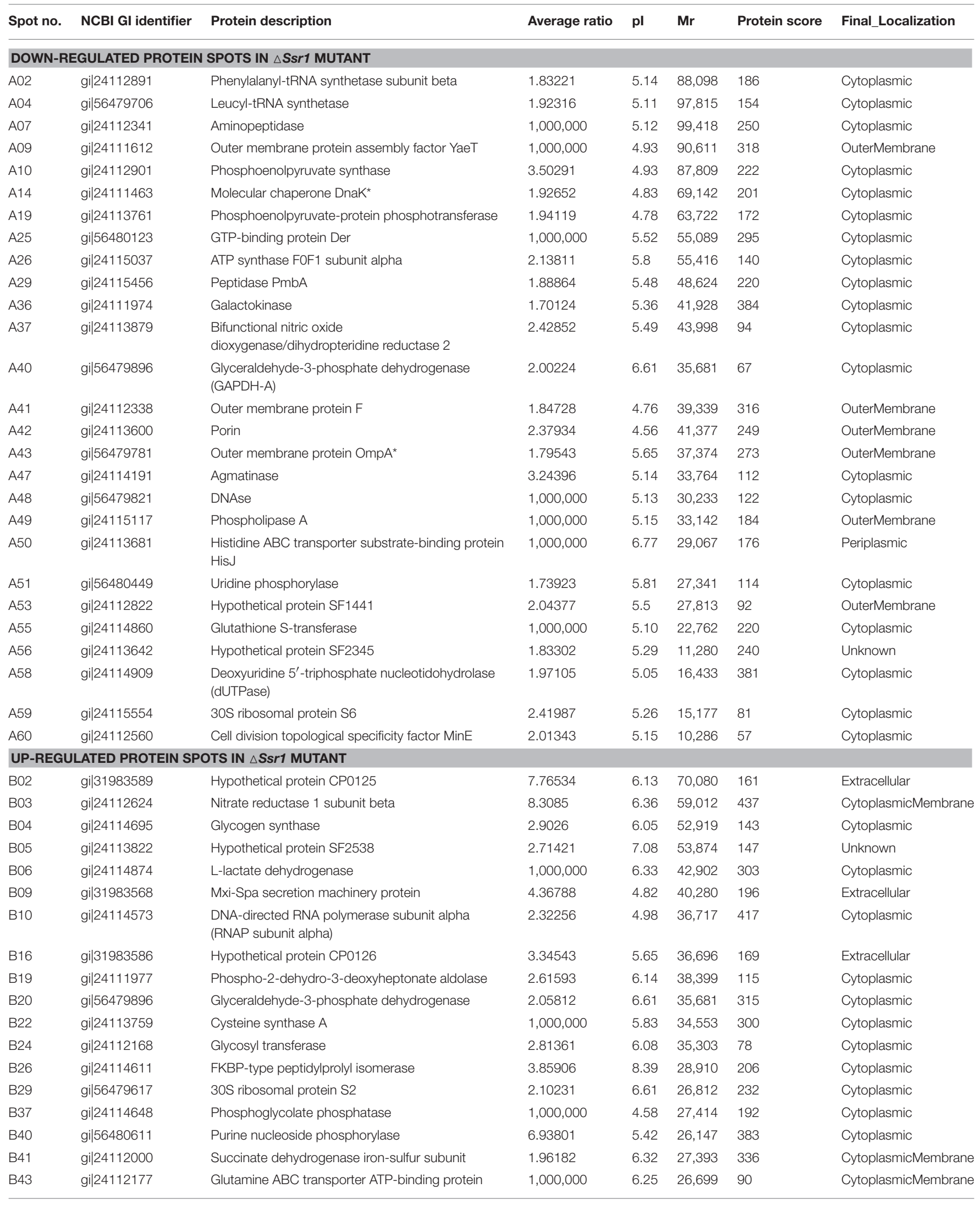


TABLE 3 | Continued

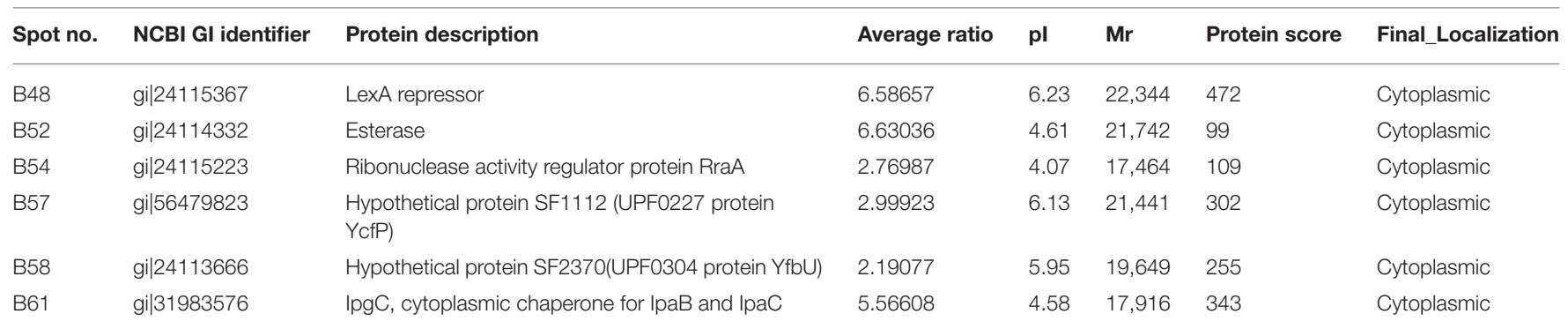

${ }^{*}$ Represent the potential targets of Ssr1, which were predicted by sTarPicker method.

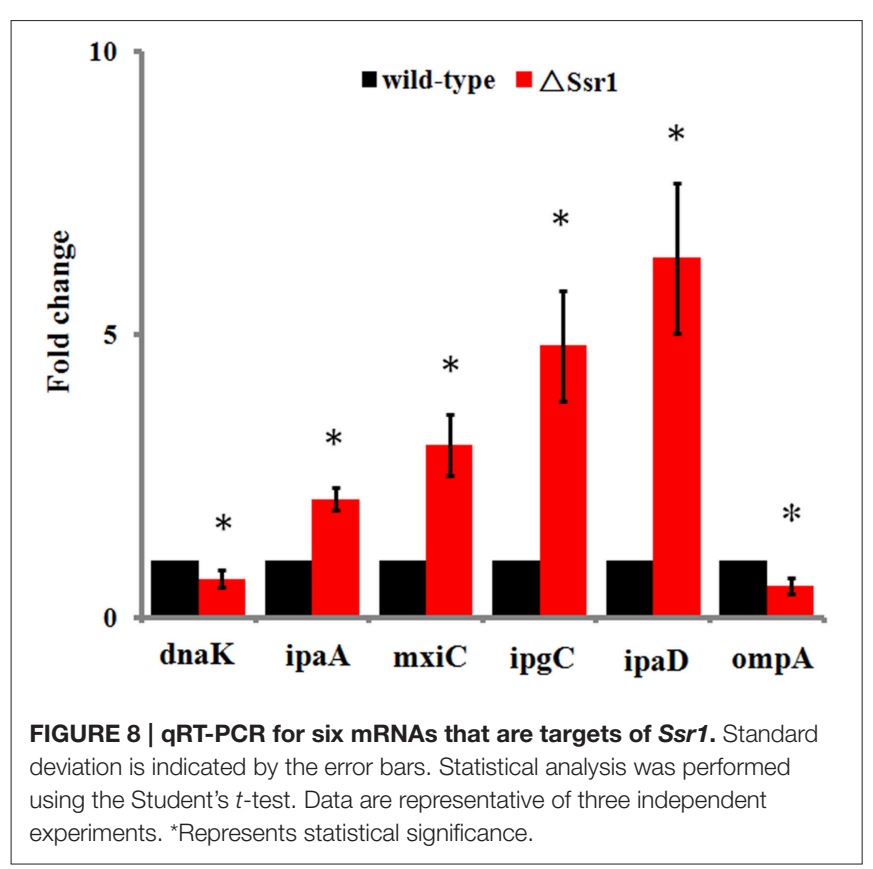

The transcriptional levels of these genes in the mutant were calculated relative to those in the wild type. Accordingly, the levels of these genes in the wild type were set to 1 as reference (Figure 8). The results were consistent with those of the 2-DE analysis, suggesting that Ssr1 modulation of stress resistance and virulence occurs in part by regulation of these proteins. By integrating the results of the sTarPicker and 2-DE analysis, 14 mRNAs (pheT, pepN, ppsA, dnaK, ptsI, engA, galK, ompA, ycfH, $y i b F, d u t$, minE, $r p s B$, and $y c f P$ ) may be direct targets of Ssr1. Our results provide evidence that Ssr1 in S. flexneri is involved in multiple physiological and biochemical processes, and has a particularly important role in stress response and virulence processes, via modulation of the above targets.

\section{DISCUSSION}

The attenuation of acidic stress is a key component of Shigella responses that determine its pathogenicity, since the bacterium must face the environmental conditions of the stomach prior to infecting the colon. In turn, activation of acidic stress resistance provides cross-protection against other environmental stresses, such as oxidation, osmotic pressure, and heat stress (Foster and Spector, 1995; Vandal et al., 2009). sRNAs are involved in the toleration of environmental stresses, and thereby contribute to the virulence of several pathogens (Marteyn et al., 2012). Here, we demonstrate that the sRNA Ssr1 play critical roles in responding to acidic stress tolerance and virulence in S. flexneri. To our knowledge, this study provides the first functional bioinformatics and wet-lab analysis of a novel sRNA (Ssr1) in S. flexneri. Ssr1 was highly expressed across a range of $\mathrm{pH}$ values, and the growth and survival of $\Delta S s r 1$ was reduced under acidic stress conditions when compared with the wild-type strain. Ssrl is strongly upregulated during acidic stress, which leads to increased tolerance; this comes at the expense of reducing the virulence and pathogenicity of Shigella.

To adapt and survive in a complex hostile environment, bacteria have to adjust their gene expression levels through regulatory networks, and this process affects the host infection process, which is particularly important for pathogenesis (Papenfort and Vogel, 2010). To fully understand the function of $S s r 1$ in both the stress response and virulence, the regulatory pathway of regulators of $S s r 1$ must be identified. Here, we found that DnaK, a member of the heat shock protein 70 (HSP70) family that assists in the refolding and hydrolysis of abnormal proteins (Zhang et al., 2014), was upregulated by Ssr 1 based on the results of qRT-PCR and proteomic analysis. The expression of HSP70 family members is increased following exposure to stress, including that induced by $\mathrm{pH}$ changes (Tomoyasu et al., 2012). Remarkably, in S. flexneri, acidic stress induced robust expression of $S s r 1$, which then may directly upregulated DnaK protein; this reveals a mechanism by which $S$. flexneri produces a stress response protein in order to survive in acidic conditions. The proteomics results revealed that Ssr1 negatively regulates the ipa and mxi genes of the T3SS system, which is required for the invasion of the colorectal epithelium and for promoting virulence in S. flexneri. However, these T3SS-related genes were predicted as indirect targets of Ssr1 by sTarPicker, implying that the precise virulence mechanism mediated by Ssr 1 requires further clarification. We suggest that regulation of E. coli OmpA, an outer membrane protein that mediates a wide-range of activities including resistance to complement, and invasion and survival within host cell, is a candidate mechanism. OmpA can 
regulate T3SS to influence virulence in Yersinia (Bartra et al., 2012). In addition, ompA expression is increased by Ssr 1 in S. flexneri, and we demonstrated that this contributes to the negative regulation of T3SS genes. Thus, we conclude that Ssr1 regulation enables a rapid response to various environmental conditions, yet can also decrease the expression of T3SS in S. flexneri. And, once the bacteria enter normal conditions, Ssr1 becomes refractory to downregulation, which leads to increased T3SS activation. The identities of all the molecular components of pathways used by bacteria to improve their tolerance while reducing their virulence under extreme environments are still unclear.

Our study of Ssr1 provides new insights into the interactions between enteric bacteria and the host environment. Although we did not validate all of the genes we identified as targets that participate in the regulatory network, our results indicate that Ssr1 sRNAs have roles in regulating genes involved in virulence and stress tolerance, specifically in response to acidic stress. These findings will help us better understand how this bacterium responds to and regulates pathogenicity under diverse environmental stress conditions. Our report also enhances the understanding of the virulence mechanisms employed by $S$. flexneri, and reaffirms the concept that bacteria use multiple strategies to modulate their pathogenesis in order to survive and thrive. However, additional evidence is needed to confirm the relationship between the stress response, stress tolerance, and virulence. In vivo experiments that address how the role of Ssr1 affects virulence during acid stress will be particularly important. We have planned further functional and mechanistic studies of sRNA regulatory networks in S. flexneri, in order to determine

\section{REFERENCES}

Bartra, S. S., Gong, X., Lorica, C. D., Jain, C., Nair, M. K., Schifferli, D., et al. (2012). The outer membrane protein A (OmpA) of Yersinia pestis promotes intracellular survival and virulence in mice. Microb. Pathog. 52, 41-46. doi: 10.1016/j.micpath.2011.09.009

Berg, O. G., and von Hippel, P. H. (1987). Selection of DNA binding sites by regulatory proteins. Statistical-mechanical theory and application to operators and promoters. J. Mol. Biol. 193, 723-750. doi: 10.1016/0022-2836(87)90354-8

Cheng, F., Wang, J., Peng, J., Yang, J., Fu, H., Zhang, X., et al. (2007). Gene expression profiling of the $\mathrm{pH}$ response in Shigella flexneri 2a. FEMS Microbiol. Lett. 270, 12-20. doi: 10.1111/j.1574-6968.2007.00647.x

Foster, J. W., and Spector, M. P. (1995). How Salmonella survive against the odds. Annu. Rev. Microbiol. 49, 145-174. doi: 10.1146/annurev.mi.49.100195.001045

Gripenland, J., Netterling, S., Loh, E., Tiensuu, T., and Toledo-Arana, A. (2010). RNAs: regulators of bacterial virulence. Nat. Rev. Microbiol. 8, 857-866. doi: $10.1038 /$ nrmicro2457

Hoe, C. H., Raabe, C. A., Rozhdestvensky, T. S., and Tang, T. H. (2013). Bacterial sRNAs: regulation in stress. Int. J. Med. Microbiol. 303, 217-229. doi: 10.1016/j.ijmm.2013.04.002

Lesnik, E. A., Sampath, R., Levene, H. B., Henderson, T. J., McNeil, J. A., and Ecker, D. J. (2001). Prediction of rho-independent transcriptional terminators in Escherichia coli. Nucleic Acids Res. 29, 3583-3594. doi: 10.1093/nar/29.17.3583

Li, G., Chen, J., Xie, P., Jiang, Y., Wu, L., and Zhang, X. (2011). Protein expression profiling in the zebrafish (Danio rerio) embryos exposed to the microcystin-LR. Proteomics 11, 2003-2018. doi: 10.1002/pmic.201000442

Livny, J., Fogel, M. A., Davis, B. M., and Waldor, M. K. (2005). sRNAPredict: an integrative computational approach to identify sRNAs in bacterial genomes. Nucleic Acids Res. 33, 4096-4105. doi: 10.1093/nar/gki715 how their interactions affect $S$. flexneri infections. Through such studies, novel approaches to treat and control S. flexneri outbreaks, such as by targeting acidic storage, may be identified. Finally, we note that our bioinformatics sRNA prediction method can also be used to identify virulence regulators in other bacteria, and may thus be a powerful tool for this research community.

\section{AUTHOR CONTRIBUTIONS}

HS, LW, and WL designed the research, assessed and interpreted the results, and prepared the manuscript. LW, GY, LQ, SQ, PL, $\mathrm{RH}, \mathrm{LJ}, \mathrm{ZW}$, and XD carried out the data analysis and designed experiments. GY, XL, JX, and LW assisted in the experiments.

\section{ACKNOWLEDGMENTS}

We are grateful to Dr. John D. Klena (United States Centers for Disease Control and Prevention, International Emerging Infections Program, Beijing, China) for critical review of the manuscript. This study was supported by the Natural Science Foundation of China (31000589) and the China Special Grant for the Prevention and Control of Infectious Diseases (2013ZX10004607).

\section{SUPPLEMENTARY MATERIAL}

The Supplementary Material for this article can be found online at: http://journal.frontiersin.org/article/10.3389/fcimb. 2016.00024
Marteyn, B., Gazi, A., and Sansonetti, P. (2012). Shigella: a model of virulence regulation in vivo. Gut Microbe. 3, 104-120. doi: 10.4161/gmic.19325

Michaux, C., Hartke, A., Martini, C., Reiss, S., Albrecht, D., Budin-Verneuil, A., et al. (2014). Involvement of Enterococcus faecalis small RNAs in stress response and virulence. Infect. Immun. 82, 3599-3611. doi: 10.1128/IAI.01900-14

Murphy, E. R., and Payne, S. M. (2007). RyhB, an iron-responsive small RNA molecule, regulates Shigella dysenteriae virulence. Infect. Immun. 75, 3470-3477. doi: 10.1128/IAI.00112-07

Opdyke, J. A., Fozo, E. M., Hemm, M. R., and Storz, G. (2011). RNase III participates in GadY-dependent cleavage of the gadX-gadW mRNA. J. Mol. Biol. 406, 29-43. doi: 10.1016/j.jmb.2010.12.009

Papenfort, K., and Vogel, J. (2010). Regulatory RNA in bacterial pathogens. Cell Host Microbe. 8, 116-127. doi: 10.1016/j.chom.2010.06.008

Papenfort, K., and Vogel, J. (2014). Small RNA functions in carbon metabolism and virulence of enteric pathogens. Front. Cell. Infect. Microbiol. 4:91. doi: 10.3389/fcimb.2014.00091

Park, S. H., Oh, K. H., and Kim, C. K. (2001). Adaptive and cross-protective responses of Pseudomonas sp. DJ-12 to several aromatics and other stress shocks. Curr. Microbiol. 43, 176-181. doi: 10.1007/s002840010283

Peng, J., Yang, J., and Jin, Q. (2011). An integrated approach for finding overlooked genes in Shigella. PLoS ONE 6:e18509. doi: 10.1371/journal.pone.0018509

Sereny, B. (1955). Experimental Shigella keratoconjunctivitis; a preliminary report. Acta Microbiol. Acad. Sci. Hung. 2, 293-296.

Shi, X., Yeung, L. W., Lam, P. K., Wu, R. S. S., and Zhou, B. S. (2009). Protein profiles in zebrafish (Danio rerio) embryos exposed to perfluorooctane sulfonate. Toxicol. Sci. 110, 334-340. doi: 10.1093/toxsci/kfp111

Storz, G., Opdyke, J. A., and Wassarman, K.M. (2006). Regulating bacterial transcription with small RNAs. Cold Spring Harb. Symp. Quant. Biol. 71, 269-273. doi: 10.1101/sqb.2006.71.033 
Teixeira-Gomes, A. P., Cloeckaert, A., and Zygmunt, M. S. (2000). Characterization of heat, oxidative, and acid stress responses in Brucella melitensis. Infect. Immun. 68, 2954-2961. doi: 10.1128/IAI.68.5.29542961.2000

Toledo-Arana, A., Repoila, F., and Cossart, P. (2007). Small noncoding RNAs controlling pathogenesis. Curr. Opin. Microbiol. 10, 182-188. doi: 10.1016/j.mib.2007.03.004

Tomoyasu, T., Tabata, A., Imaki, H., Tsuruno, K., Miyazaki, A., Sonomoto, K., et al. (2012). Role of Streptococcus intermedius DnaK chaperone system in stress tolerance and pathogenicity. Cell Stress Chaperones. 17, 41-55. doi: 10.1007/s12192-011-0284-4

Vandal, O. H., Nathan, C. F., and Ehrt, S. (2009). Acid resistance in Mycobacterium tuberculosis. J. Bacteriol. 191, 4714-4721. doi: 10.1128/JB.00305-09

Vogel, J. (2009). A rough guide to the non-coding RNA world of Salmonella. Mol. Microbiol. 71, 1-11. doi: 10.1111/j.1365-2958.2008.06505.x

Ying, X., Cao, Y., Wu, J., Liu, Q., Cha, L., and Li, W. (2011). sTarPicker: a method for efficient prediction of bacterial sRNA targets based on a twostep model for hybridization. PLoS ONE 6:e22705. doi: 10.1371/journal.pone. 0022705
Zhang, H., Jiang, X., Xiao, W., and Lu, L. (2014). Proteomic strategy for the analysis of the polychlorobiphenyl-degrading cyanobacterium Anabaena PD-1 exposed to Aroclor 1254. PLoS ONE 9:e91162. doi: 10.1371/journal.pone.0091162

Zhou, D. H., Yuan, Z. G., Zhao, F. R., Li, H. L., Zhou, Y., Lin, R. Q., et al. (2011). Modulation of mouse macrophage proteome induced by Toxoplasma gondii tachyzoites in vivo. Parasitol. Res. 109, 1637-1646. doi: 10.1007/s00436-0112435-z

Conflict of Interest Statement: The authors declare that the research was conducted in the absence of any commercial or financial relationships that could be construed as a potential conflict of interest.

Copyright (C) 2016 Wang, Yang, Qi, Li, Jia, Xie, Qiu, Li, Hao, Wu, Du, Li and Song. This is an open-access article distributed under the terms of the Creative Commons Attribution License (CC BY). The use, distribution or reproduction in other forums is permitted, provided the original author(s) or licensor are credited and that the original publication in this journal is cited, in accordance with accepted academic practice. No use, distribution or reproduction is permitted which does not comply with these terms. 